\title{
Dirac Quantization of the Chern-Simons Field Theory in the Coulomb Gauge
}

\author{
F. Ferrari* ${ }^{a, b}$ and I. Lazzizzera ${ }^{a, c}$ \\ a Dipartimento di Fisica, Universitá di Trento, 38050 Povo (TN), Italy \\ ${ }^{b}$ LPTHE $^{\dagger}$, Université Pierre er Marie Curie-PARIS VI, Université Denis Diderot-Paris VII, \\ Boite 126, Tour 16, $1^{\text {er }}$ étage, 4 place Jussieu, F-75252 Paris CEDEX 05, FRANCE \\ c INFN, Gruppo Collegato di Trento, Italy
}

(September 1996)

\begin{abstract}
In this letter the Chern-Simons field theories are studied in the Coulomb gauge using the Dirac's canonical formalism for constrained systems. As a strategy, we first work out the constraints and then quantize, replacing the Dirac brackets with quantum commutators. We find that the Chern-Simons field theories become two dimensional models with no propagation along the time direction. Moreover, we prove that, despite of the presence of non-trivial self-interactions in the gauge fixed functional, the commutation relations between the fields are trivial at any order in perturbation theory in the absence of couplings with matter fields. If these couplings are present, instead, the commutation relations become rather involved, but it is still possible to study their main properties and to show that they vanish at the tree level.
\end{abstract}

\footnotetext{
*The work of F. Ferrari has been supported in part by the European Union, TMR Programme, under grant ERB4001GT951315

†Laboratoire associé No. 280 au CNRS
} 


\section{INTRODUCTION}

In this letter we investigate the Chern-Simons (C-S) field theories [1 3 with gauge group $S U(n)$ in the Coulomb gauge using the Dirac's formalism for constrained systems [4.5]. As it happens in the case of the more popular covariant gauges, also in this gauge the C-S functional contains self-interactions, but the Feynman rules simplify considerably and can be explicitly derived even on space-times with a non-flat spatial section [6]. Another advantage of the Coulomb gauge is that there are no time derivatives in the gauge fixed action, so that the $\mathrm{C}-\mathrm{S}$ theory becomes in practice a two-dimensional model. Despite of many physical and mathematical applications of the $\mathrm{C}-\mathrm{S}$ field theories [3, [7], however, until now only a few calculations have been performed in the Coulomb gauge [6, 8, 9]. One of the main reasons is that, already in the case of the Yang-Mills field theories, several perplexities arise concerning the use of this gauge 11 13. Analogous problems are unfortunately present also in $\mathrm{C}-\mathrm{S}$ field theories, but in a milder form, so that these models provide an important laboratory in order to study the possible remedies. For example, in the abelian case it is known that the so-called Maxwell-Chern-Simons (MCS) theory is affected by the presence of infrared divergences in the Coulomb gauge [1,2]. Nevertheless, it has recently been shown in ref. [9] that the theory can be consistently worked out and that for instance the Möller scattering amplitudes computed in the Coulomb gauge and in the covariant gauges coincide at all orders in perturbation theory. Other tests confirming the safety of the Coulomb gauge in the MCS models can also be found in [9].

On the other side, the ambiguities in the Yang-Mills Feynman integrals pointed out in [11] arise as well in the nonabelian $\mathrm{C}-\mathrm{S}$ field theories due to the absence of time derivatives in the action [6]. A simple recipe to regularize such ambiguities has been proposed and successfully tested in first order calculations [6]. However, a detailed investigation of the consistency of the nonabelian $\mathrm{C}-\mathrm{S}$ field theories in the Coulomb gauge at any perturbative

order is still missing. As one of the steps to fill this gap, we exploit in this letter the formalism of Dirac's canonical approach to constrained systems [⿶, 5]. 
We notice that, besides some subtleties already noticed in [16], the derivation of the final Dirac brackets requires in the Coulomb gauge some care with distributions. Moreover, the final commutation relations (CR's) between the fields obtained here are rather involved. At a first sight, this is surprising in topological field theories with vanishing Hamiltonian and without degrees of freedom. However, at least in the case considered here, in which there are no interactions with matter fields, we show that this contradiction is only apparent. As a matter of fact, taking into account the Gauss law and the Coulomb gauge fixing, it can be proved that the commutation relations between the gauge fields vanish identically at any perturbative order as expected. In this way we discover that the Chern-Simons field theories in the Coulomb gauge are not only perturbatively finite as has been checked in the covariant gauges [14], but also free. This is not a priori evident, because in the Coulomb gauge the C-S functional contains non-trivial self-interaction terms.

The material presented in this paper is divided as follows. In the next Section we will present our results. In the Conclusions we will discuss the open problems and the possible further developments.

\section{CANONICAL QUANTIZATION OF THE C-S FIELD THEORY IN THE COULOMB GAUGE}

The Lagrangian of the pure $S U(N)$ Chern-Simons $(\mathrm{C}-\mathrm{S})$ field theory in three dimensions is given by

$$
L_{C S}=\frac{s}{8 \pi} \epsilon^{\mu \nu \rho}\left(A_{\mu}^{a} \partial_{\nu} A_{\rho}^{a}-\frac{1}{3} f^{a b c} A_{\mu}^{a} A_{\nu}^{b} A_{\rho}^{c}\right)
$$

where $s$ is a dimensionless coupling constant and $A_{\mu}^{a}$ is the gauge potential. Greek letters $\mu, \nu, \rho, \ldots=0,1,2$ will denote space-time indices while the first latin letters $a, b, c, \ldots=$ $1, \cdots, N$ will denote color indices. Moreover, the totally antisymmetric tensor $\epsilon^{\mu \nu \rho}$ is defined by the convention $\epsilon^{012}=1$. Finally, a Minkowski metric $g_{\mu \nu}=\operatorname{diag}(1,-1,-1)$ will be 
assumed. To derive the $\mathrm{C}-\mathrm{S}$ Hamiltonian, we have to compute the canonical momenta:

$$
\pi^{\mu, a}(\mathbf{x}, t)=\frac{\delta S_{C S}}{\delta\left(\partial_{0} A_{\mu}(\mathbf{x}, t)\right)}
$$

Here we have put $S_{C S}=\int d^{3} x L_{C S}, t=x^{0}$ and $\mathbf{x}=\left(x^{1}, x^{2}\right)$.

The nonvanishing Poisson brackets (PB) among canonical variables are:

$$
\left\{A_{\mu}^{a}(\mathbf{x}, t), \pi_{\nu}^{b}(\mathbf{y}, t)\right\}=\delta^{a b} g_{\mu \nu} \delta^{(2)}(\mathbf{x}-\mathbf{y})
$$

From eqs. (11) and (2) we obtain:

$$
\pi^{0, a}=0 \quad \pi^{i, a}=\frac{s}{8 \pi} \epsilon^{i j} A_{j}^{a}
$$

where $\epsilon^{i j}, i, j=1,2$, is the two dimensional totally antisymmetric tensor satisfying the definition $\epsilon^{12}=1$. A straightforward calculation shows that the $\mathrm{C}-\mathrm{S}$ Hamiltonian is given by:

$$
H_{C S}=-\int d^{2} \mathbf{x} A_{0}^{a}\left(D_{i}^{a b} \pi^{i, b}+\partial_{i} \pi^{i, a}\right)
$$

In the above equation $D_{i}^{a b}$ denotes the spatial components of the covariant derivative:

$$
D_{\mu}^{a b} \equiv \partial_{\mu} \delta^{a b}+f^{a b c} A_{\mu}^{c}
$$

From eqs. (3) we obtain the constraints:

$$
\begin{aligned}
& \varphi^{0, a}=\pi^{0, a} \\
& \varphi^{i, a}=\pi^{i, a}-\frac{s}{8 \pi} \epsilon^{i j} A_{j}^{a} \quad i=1,2
\end{aligned}
$$

Following the Dirac procedure for constrained systems, the latter will be imposed in the weak sense:

$$
\varphi^{\mu, a} \approx 0
$$

To this purpose, we construct the extended Hamiltonian:

$$
\widetilde{H}=H_{C S}+\int \lambda_{\mu}^{a} \varphi^{\mu, a} d^{2} \mathbf{x}
$$


where the $\lambda_{\mu}^{a}$ 's represent the Lagrange multipliers corresponding to the primary constraints $\varphi^{\mu, a}$.

From the consistency conditions $\dot{\varphi}^{\mu, a}=\left\{\varphi^{\mu, a}, \widetilde{H}_{C S}\right\} \approx 0$, we obtain the secondary constraint:

$$
\mathcal{G}^{a}=D_{i}^{a b} \pi^{i, b}+\partial_{i} \pi^{i, b} \approx 0 \quad \text { Gauss law }
$$

and two relations which determine the Lagrange multipliers $\lambda_{1}$ and $\lambda_{2}$ :

$$
\frac{s}{4 \pi} \epsilon^{i j}\left(D_{j}^{a b} A_{0}^{b}-\lambda_{j}^{a}\right) \approx 0 \quad i=1,2
$$

It is possible to see that the consistency condition $\dot{\mathcal{G}}^{a} \approx 0$ does not lead to any further independent equation. Let us notice that the operators $\mathcal{G}^{a}$ generate the $S U(N)$ group of gauge transformations. To show this, we introduce the Dirac brackets (DB's) associated to the second class constraints $\varphi_{i}^{a}$ of eq. (6):

$$
\{A(\mathbf{x}), B(\mathbf{y})\}^{*}=\{A(\mathbf{x}), B(\mathbf{y})\}-\sum_{i, j=1}^{2} \int d^{2} \mathbf{x}^{\prime} d^{2} \mathbf{y}^{\prime}\left\{A(\mathbf{x}), \varphi^{i}\left(\mathbf{x}^{\prime}\right)\right\}\left(C^{-1}\right)_{i j}\left(\mathbf{x}^{\prime}, \mathbf{y}^{\prime}\right)\left\{\varphi^{j}\left(\mathbf{y}^{\prime}\right), B(\mathbf{y})\right\}
$$

where $\left(C^{-1}\right)_{i j}(\mathbf{x}, \mathbf{y})$ is the inverse of the matrix $C^{i j}(\mathbf{x}, \mathbf{y})=\left\{\varphi^{i}(\mathbf{x}), \varphi^{j}(\mathbf{y})\right\}$. For simplicity, color indices and the time variable have been omitted in the above equations.

Computing $\left(C^{-1}\right)_{i j}(\mathbf{x}, \mathbf{y})$ explicitly, we find:

$$
\left(C^{-1}\right)_{i j}^{a b}(\mathbf{x}, \mathbf{y})=\frac{4 \pi}{s} \delta^{a b} \epsilon_{i j} \delta(\mathbf{x}-\mathbf{y})
$$

The Dirac brackets among the canonical variables are now given by

$$
\begin{gathered}
\left\{A_{i}^{a}(t, \mathbf{x}), \pi^{j, b}(t, \mathbf{y})\right\}^{*}=\frac{1}{2} \delta^{a b} \delta_{i}^{j} \delta(\mathbf{x}-\mathbf{y}) \\
\left\{A_{i}^{a}(t, \mathbf{x}), A_{j}^{b}(t, \mathbf{y})\right\}^{*}=\frac{4 \pi}{s} \delta^{a b} \epsilon_{i j} \delta(\mathbf{x}-\mathbf{y}) \\
\left\{\pi^{i, a}(t, \mathbf{x}), \pi^{j, b}(t, \mathbf{y})\right\}^{*}=\frac{s}{16 \pi} \delta^{a b} \epsilon^{i j} \delta(\mathbf{x}-\mathbf{y})
\end{gathered}
$$

In the following, the DB's defined in (10) will be written without the superscript * . Exploiting the DB's (11)-(13), we obtain the relations: 


$$
\begin{aligned}
\left\{\mathcal{G}^{a}(t, \mathbf{x}), A_{i}^{b}(t, \mathbf{y})\right\} & =-D_{i}^{a b}(x) \delta(\mathbf{x}-\mathbf{y}) \\
\left\{\mathcal{G}[\psi], A_{i}^{a}(t, \mathbf{x})\right\} & =D_{i}^{a b}(x) \psi^{b}(\mathbf{x}) \\
\left\{\mathcal{G}^{a}(t, \mathbf{x}), \mathcal{G}^{b}(t, \mathbf{y})\right\} & =-f^{a b c} \mathcal{G}^{c}(t, \mathbf{x}) \delta(\mathbf{x}-\mathbf{y})
\end{aligned}
$$

where $\mathcal{G}[\psi]=\int d^{2} \mathbf{x} G^{a}(t, \mathbf{x}) \psi^{a}(\mathbf{x})$. This shows that the $\mathcal{G}^{a}(t, \mathbf{x})$ are the generators of the $S U(N)$ gauge transformations as desired.

At this point, we are left with the constraints given by eq. (5) and by the Gauss law (8). However, the former constraint, which involves the conjugate momentum of $A_{0}^{a}$ can be ignored. As a matter of fact, the field $A_{0}^{a}$ plays just the role of the Lagrange multiplier associated to the Gauss law in the Hamiltonian (四) and has no dynamics. From eqs. (14)(16) it turns out that the Gauss law (8) is a first class constraint. To make it second class, we introduce the Coulomb gauge fixing:

$$
\partial_{i} A^{i, a} \approx 0
$$

and the new extended Hamiltonian:

$$
\check{H}_{C S}=\int d^{2} \mathbf{x}\left[-A_{0}^{a} \mathcal{G}^{a}+\frac{s}{8 \pi} A_{i}^{a} \partial^{i} B^{a}+\lambda_{0}^{a} \pi^{0, a}\right]
$$

From the condition $\left\{\partial_{i} A^{i, a}, \check{H}_{C S}\right\} \approx 0$, we obtain an equation for $A_{0}^{a}$ :

$$
\partial^{i} D_{i}^{a b} A_{0}^{b} \approx 0
$$

Moreover, the requirement $\left\{\partial^{i} D_{i}^{a b} A_{0}^{b}(x), \check{H}_{C S}\right\} \approx 0$ determines the Lagrange multiplier $\lambda_{0}$ :

$$
-\triangle \lambda_{0}^{a}-\left\{\partial_{i}\left(\mathbf{A}_{i} \times \mathbf{A}_{0}\right)^{a}, \check{H}_{C S}\right\} \approx 0
$$

In the above equation the symbol $\triangle$ denotes the two dimensional Laplacian $\triangle=-\partial_{i} \partial^{i}$ and

$$
\left(\mathbf{A}_{i} \times \mathbf{A}_{0}\right)^{a} \equiv f^{a b c} A_{i}^{b} A_{0}^{c}
$$

Another independent equation, which fixes the Lagrange multipliers $B^{a}$, is provided by the requirement $\dot{\mathcal{G}}^{a} \approx 0$ : 


$$
\left\{\mathcal{G}^{a}, \check{H}_{C S}\right\} \approx-\frac{s}{8 \pi} D_{i}^{a b} \partial^{i} B^{b} \approx 0
$$

Let us notice that the above relations (8), (17) and (19)-(21) are compatible with the equations of motion of the gauge potentials:

$$
\begin{gathered}
\epsilon^{i j}\left(D_{i}^{a b} A_{j}^{b}-\partial_{j} A_{i}^{a}\right)=0 \\
D_{j}^{a b} A_{0}^{b}-\partial_{0} A_{j}^{a}=0
\end{gathered}
$$

As a matter of fact (22) is equivalent to the condition $\mathcal{G}^{a}=0$. Moreover, multiplying for instance eq. (23) with the differential operator $\epsilon_{k i} \partial^{k}$, we obtain the relation:

$$
\partial_{0} \partial^{k} A_{k}^{a}-\partial^{k} D_{k}^{a b} A_{0}^{b}=0
$$

which is consistent with the Coulomb gauge and the condition (19) on $A_{0}^{a}$.

It is now possible to realize that the Gauss law (8) and the Coulomb gauge fixing (17) form a set of second class constraints, so that we can impose them in the strong sense computing the final Dirac brackets. Putting

$$
\chi_{1}^{a}=\mathcal{G}^{a} \quad \chi_{2}^{a}=\partial_{i} A^{i, a}
$$

with $\alpha, \beta=1,2$, we have for any two observables $A(\mathbf{x})$ and $B(\mathbf{y})$ :

$$
\begin{gathered}
\left\{A^{a}(\mathbf{x}), B^{b}(\mathbf{y})\right\}^{*}=\left\{A^{a}(\mathbf{x}), B^{b}(\mathbf{y})\right\}- \\
\sum_{\alpha, \beta=1}^{2} \sum_{c, d} \int d^{2} \mathbf{x}^{\prime} d^{2} \mathbf{y}^{\prime}\left\{A^{a}(\mathbf{x}), \chi_{\alpha}^{c}\left(\mathbf{x}^{\prime}\right)\right\}\left(C^{-1}\right)^{\alpha \beta, c d}\left(\mathbf{x}^{\prime}, \mathbf{y}^{\prime}\right)\left\{\chi^{\beta, d}\left(\mathbf{y}^{\prime}\right), B^{b}(\mathbf{y})\right\}
\end{gathered}
$$

The matrix $\left(C^{-1}\right)^{\alpha \beta, c d}(\mathbf{x}, \mathbf{y})$ denotes the inverse of the $2 \times 2$ matrix $C_{\alpha \beta}^{a b}(\mathbf{x}, \mathbf{y})=$ $\left\{\chi_{\alpha}^{a}(\mathbf{x}), \chi_{\beta}^{b}(\mathbf{y})\right\}$. After some manipulations and remembering that the gauge potentials satisfy the Coulomb gauge constraint, we obtain:

\footnotetext{
${ }^{1}$ In the following, the time variable will be omitted from our equations.
} 


$$
\mathbf{C}^{a b}(\mathbf{x}, \mathbf{y})=\left(\begin{array}{cc}
0 & -D_{i}^{a b}(\mathbf{x}) \partial_{\mathbf{x}}^{i} \delta(\mathbf{x}-\mathbf{y}) \\
D_{i}^{a b}(\mathbf{x}) \partial_{\mathbf{x}}^{i} \delta(\mathbf{x}-\mathbf{y}) & 0
\end{array}\right)
$$

To invert the above matrix, it is convenient to introduce the function $\mathcal{D}^{c b}(\mathbf{x}, \mathbf{y})$, defined by the following equation [15]:

$$
D_{i}^{a c}(\mathbf{x}) \partial_{\mathbf{x}}^{i} \mathcal{D}^{c b}(\mathbf{x}, \mathbf{y})=\delta^{a b} \delta(\mathbf{x}-\mathbf{y})
$$

Supposing that the Green function $\mathcal{D}^{a b}(\mathbf{x}, \mathbf{y})$ has a sufficiently good behavior at infinity, it is easy to prove that

$$
\left(\mathbf{C}^{-1}\right)^{a b}(\mathbf{x}, \mathbf{y})=\left(\begin{array}{cc}
0 & \mathcal{D}^{a b}(\mathbf{x}, \mathbf{y}) \\
-\mathcal{D}^{a b}(\mathbf{x}, \mathbf{y}) & 0
\end{array}\right)
$$

After imposing the constraints (8) and (17) in the strong sense, the Hamiltonian $\check{H}_{C S}$ vanishes, but the commutation relations (CR's) between the fields remain complicated. From eqs. (24) and (26), in fact, the basic DB's between the canonical variables $A_{i}^{a}$ have the following form:

$$
\begin{gathered}
\left\{A_{i}^{a}(\mathbf{x}), A_{j}^{b}(\mathbf{y})\right\}^{*}=-\frac{4 \pi}{s} \delta^{a b} \epsilon_{i j} \delta(\mathbf{x}-\mathbf{y})+ \\
\frac{4 \pi}{s} \epsilon_{i k} \partial_{\mathbf{x}}^{k} D_{j}^{b c}(\mathbf{y}) \mathcal{D}^{a c}(\mathbf{x}, \mathbf{y})-\frac{4 \pi}{s} \epsilon_{k j} D_{i}^{a c}(\mathbf{x}) \partial_{\mathbf{y}}^{k} \mathcal{D}^{c b}(\mathbf{x}, \mathbf{y})
\end{gathered}
$$

Let us study the main properties of the above DB's. First of all, they are antisymmetric as expected:

$$
\left\{A_{i}^{a}(\mathbf{x}), A_{j}^{b}(\mathbf{y})\right\}^{*}=-\left\{A_{j}^{b}(\mathbf{y}), A_{i}^{a}(\mathbf{x})\right\}^{*}
$$

The antisymmetry of the right hand side of eq. (27) is not explicit, but can be verified with the help of the relation:

$$
\mathcal{D}^{a b}(\mathbf{x}, \mathbf{y})=\mathcal{D}^{b a}(\mathbf{y}, \mathbf{x})
$$


The above symmetry of the Green function $\mathcal{D}^{a b}(\mathbf{x}, \mathbf{y})$ in its arguments is a consequence of the selfadjointness of the defining equation (25) [15]. Moreover, the CR's (27) are consistent with the Coulomb gauge. As a matter of fact, it is easy to prove that:

$$
\left\{A_{i}^{a}(\mathbf{x}), \partial^{j} A_{j}^{b}(\mathbf{y})\right\}^{*}=\left\{\partial^{i} A_{i}^{a}(\mathbf{x}), A_{j}^{b}(\mathbf{y})\right\}^{*}=0
$$

The case of a Chern-Simons field theory with abelian gauge group $U(1)$ is particularly instructive in order to understand the meaning of the CR's (27). Let $U_{\mu}$ denote the abelian gauge fields. Then the Lagrangian (1) reads:

$$
L_{C S}=\frac{s}{8 \pi} \epsilon^{\mu \nu \rho} U_{\mu} \partial_{\nu} U_{\rho}
$$

It is now possible to decompose the gauge potentials $U_{i}, i=1,2$ into transverse and longitudinal components:

$$
U_{i}=\epsilon^{i j} \partial_{j} \varphi+\partial_{i} \rho
$$

where $\varphi$ and $\rho$ are two real scalar fields. Exploiting the Coulomb gauge condition it turns out that $\rho=0$. The canonical momenta are given by:

$$
\pi^{i}=\frac{s}{8 \pi} \epsilon^{i j} U_{j}
$$

As a consequence, from the Gauss law $\partial_{i} \pi^{i}=0$, we obtain the relation $\partial_{i} \partial^{i} \varphi=0$. This implies that $\varphi=0$ and thus there is no dynamics in the $\mathrm{C}-\mathrm{S}$ field theory as expected.

The CR's (27) must be consistent with that fact. Indeed, in the abelian case it is easy to derive the Green function $\mathcal{D}(\mathbf{x}, \mathbf{y})$ solving eq. (25). The result is:

$$
\mathcal{D}(\mathbf{x}, \mathbf{y})=-\frac{1}{2 \pi} \log |\mathbf{x}-\mathbf{y}|
$$

Substituting the right hand side of the above equation in (27), we obtain:

$$
\left[U_{i}(t, \mathbf{x}), U_{j}(t, \mathbf{y})\right]=0
$$

so that the fields do not propagate as expected. To conclude the discussion of the abelian case, let us notice that eqs. (9) and (19)-(21) admit only the trivial solutions $U_{0}=\lambda_{\mu}=B=$ 
0 in agreement with the fact that, in absence of couplings with matter fields, the $\mathrm{C}-\mathrm{S}$ theory is topological and there are no degrees of freedom. In the nonabelian case the situation is analogous, but the equations of motion of the constraints become nonlinear and can in general be solved only using a perturbative approach. The relevant equations determining the fields $A_{i}^{a}(z)$, with $i=1,2$, are given by:

$$
F_{12}^{a}=\partial_{1} A_{2}^{a}-\partial_{2} A_{1}^{a}-g f^{a b c} A_{1}^{b} A_{2}^{c}
$$

and

$$
\partial_{1} A_{1}^{a}-\partial_{2}^{a} A_{2}^{a}=0
$$

With respect to eq. (11), we have introduced here the new coupling constant $g^{2}=\frac{8 \pi}{9 s}$ and the fields $A_{\mu}$ have been rescaled in such a way that the new action becomes:

$$
L=\epsilon^{\mu \nu \rho}\left(A_{\mu}^{a} \partial_{\nu} A_{\rho}^{a}-g f^{a b c} A_{\mu}^{a} A_{\nu}^{b} A_{\rho}^{c}\right)
$$

In the following, we will also suppose that $g$ is so small that a perturbative treatment of the $\mathrm{C}-\mathrm{S}$ field theory makes sense. Under this hypothesis, the fields $A_{i}^{a}$ can be expanded in powers of $g$ :

$$
A_{i}^{a}(x)=\sum_{n=0}^{\infty} g^{n} A_{i}^{a(n)}(x)
$$

where, from eqs. (31) and (32), the $A_{i}^{a(n)}$ 's satisfy the following equations:

$$
\partial_{1} A_{2}^{a(0)}-\partial_{2} A_{1}^{a(0)}=0 \quad \partial_{1} A_{1}^{a(0)}+\partial_{2} A_{2}^{a(0)}=0
$$

and

$$
\begin{array}{cr}
\partial_{1} A_{2}^{a(n)}-\partial_{2} A_{1}^{a(n)}-g f^{a b c} A_{1}^{b(n-1)} A_{2}^{c(n-1)}=0 & n=1, \ldots, \infty \\
\partial_{1} A_{1}^{a(n)}+\partial_{2} A_{2}^{a(n)}=0 & n=1, \ldots, \infty
\end{array}
$$

Assuming that the gauge fields vanish at infinity, the solution of the above equations at the zeroth order is 


$$
A_{1}^{a(0)}(t, \mathbf{x})=A_{2}^{a(0)}(t, \mathbf{x})=0
$$

as shown in the abelian case. Moreover, from eq. (33), it turns out that $A_{1}^{a(n)}(t, \mathbf{x})=0$ for $n=1, \ldots, \infty$, so that all the field configurations solving eqs. (31)-(32) vanish identically. Pure gauge solutions obtained performing gauge transformations are not allowed because, at least within perturbation theory, the Coulomb gauge fixes the gauge freedom completely. As a consequence, the right hand side of (27) is equal to zero. Indeed, due to eq. (35), the Green function $\mathcal{D}^{a b}(\mathbf{x}, \mathbf{y})$ is given by:

$$
\mathcal{D}^{a b}(\mathbf{x}, \mathbf{y})=-\delta^{a b} \frac{1}{2 \pi} \log |\mathbf{x}-\mathbf{y}|
$$

and, substituting in eq. (27), we obtain:

$$
\left\{A_{i}^{a}(\mathbf{x}), A_{j}^{b}(\mathbf{y})\right\}^{*}=0
$$

as expected.

Of course, the vanishing of the gauge fields leads to the trivial solutions $A_{0}^{a}=\lambda_{\mu}^{a}=B^{a}=0$ for the Lagrange multipliers as in the abelian case.

It is worth remarking, that the would be Poincaré algebra becomes trivial a posteriori, that is when computed on the "physical" solutions of the theory (eq. 35), characterized by the "strong" validity of the constraints and of the brackets given in eq. (37). That means that the Poincaré covariance is recovered through the trivial representation of the Poincaré group 2. We stress the fact that one must evaluate in such a posteriori way the algebra, as otherwise one finds "extra" terms, proportional to the constraints. For instance, the intermediate Dirac brackets (11 13) yield for the generators of the time and the space translations the following result:

$$
\left\{P_{0}, P_{k}\right\}=\int d^{2} x A_{0}^{a} \partial_{k} \mathcal{G}^{a}
$$

\footnotetext{
${ }^{2}$ It is worth mentioning that in the case of the Maxwell-Chern-Simons theory the Poincaré covariance takes place through a nontrivial representation of the Poincaré group [9].
} 
where $\mathcal{G}$ is given in (8). Let us notice that in the case of the MCS theory the Poncaré invariance has been proved in the Coulomb gauge within the frame of the canonical formalism in ref. [9].

To quantize the theory, we have to replace the Dirac brackets (27) with commutators. At least in the absence of coupling with matter fields, we obtain trivial commutation relations between the gauge potentials:

$$
\left[A_{i}^{a}(\mathbf{x}), A_{j}^{b}(\mathbf{y})\right]=0
$$

\section{CONCLUSIONS}

In this paper the $\mathrm{C}-\mathrm{S}$ field theories have been quantized in the Coulomb gauge within the Dirac's canonical approach to constrained systems. All the constraints coming from the Hamiltonian procedure and by the Dirac's consistency requirements have been derived. As anticipated in the Introduction, the $\mathrm{C}-\mathrm{S}$ theories become in this gauge two dimensional models. Only the fields $A_{i}^{a}$, for $i=1,2$, have in fact a dynamics, which is governed by the commutation relations (27). If no interactions with matter fields are present, we have shown that these CR's vanish at all perturbative orders. Thus the C-S field theories in the Coulomb gauge are not only finite, but also free. This result has been verified with explicit perturbative calculations of the correlation functions in [19]. A natural question that arises at this point is if analogous conclusions can be drawn for the covariant gauges. For this reason it would be interesting to repeat the procedure of canonical quantization developed here also in this case.

The situation becomes different if the interactions with other fields are switched on. Adding for instance a coupling with a current $J_{\mu}^{a}$ of the kind $\int d^{2} \mathbf{x} A_{\mu}^{a} J^{\mu, a}$ to the Hamiltonian (18), it is possible to see that the Gauss law (8) is modified as follows:

$$
D_{i}^{a b} \pi^{i, b}+\partial_{i} \pi^{i, b}+J_{0}^{a} \approx 0
$$


Thus eqs. (35) are no longer valid and we have to consider the full commutation relations (27). Remarkably, they trivially vanish at the zeroth level in the coupling constant $g$. Moreover, the CR's (27) are perfectly well defined and do not lead to ambiguities in the quantization of the $\mathrm{C}-\mathrm{S}$ models in the Coulomb gauge. In particular, we have verified here the consistency of (27) with the Coulomb gauge fixing and their antisymmetry under the exchange of the fields.

A physical application of our results, which is currently under consideration, is the investigation of the statistics of fermionic and bosonic matter fields interacting with nonabelian C-S theories at high temperatures [17]. Other interesting applications are $(2+1)$ quantum gravity and the calculation of the new link invariants from $\mathrm{C}-\mathrm{S}$ field theories quantized on Riemann surfaces, whose existence has been formally shown in [18]. In these latter two cases, the possibility offered by the Coulomb gauge of performing explicit calculations also on non-flat space-times [6] can be exploited.

[1] R. Jackiw and S. Templeton, Phys. Rev. D23 (1981), 2291; S. Deser, R. Jackiw and S. Templeton, Phys. Rev. Lett. 48 (1983), 975.

[2] C. Hagen, ibid. 157 (1984), 342; Phys. Rev. D31 (1985), 2135; J. Schonfeld, Nucl. Phys. B185 (1981), 157.

[3] E. Witten, Comm. Math. Phys. 121 (1989), 351.

[4] P. A. M. Dirac, Lectures in Quantum Mechanics, Yeshiva University Press, New York 1964.

[5] A. Hanson, T. Regge and C. Teitelboim, Constrained Hamiltonian Systems, Accademia dei Lincei, Roma, 1976 and references therein.

[6] F. Ferrari, Phys. Rev. D50 (1994), 7578.

[7] G. Moore and N. Seiberg, Phys. Lett. B220 (1989), 422; J. Fröhlich and C. King, Comm. 
Math. Phys. 126 (1989), 167; E. Guadagnini, M. Martellini and M. Mintchev, Nucl. Phys. B336 (1990), 581; G. W. Semenoff, Phys. Rev. Lett 61 (1988), 517; E. Fradkin Phys. Rev. Lett. 63 (1989), 322; M. Lüscher, Nucl. Phys. B326 (1989), 557; E. Witten, Nucl. Phys. B311 (1988), 46; Y. H. Chen, F. Wilczek, E. Witten and B. I. Halperin, Int. Jour. Mod. Phys. B3 (1989), 1001; R. Iengo and K. Lechner, Phys. Rep. 213 (1992), 179.

[8] S. Deser, R. Jackiw and S. Templeton, Ann. Phys. (N. Y.) 140 (1984), 372; A. Bellini, M. Ciafaloni and P. Valtancoli, Nucl. Phys. B454 (1995), 449; B462 (1996), 453; Phys. Lett.; K. Haller and E. L. Lombridas, Ann. Phys. 246 (1996), 1.

[9] F. P. Devecchi, M. Fleck, H. O. Girotti, M. Gomes and A. J. da Silva, Ann. Phys. 242 (1995), 275; O. Bergman and G. Lozano, Ann. Phys. (NY) 229 (1994), 229; D. Bak and O. Bergman, Phys. Rev. D51 (1995), 1994; M.-I. Park and Y.-J. Park, Phys. Rev. D50 (1994), 7584.

[10] A. Foerster and H. O. Girotti, Statistical transmutations in $2+1$ dimensions, in J. J. Giambiagi Festschrift, eds. H. Falomir, R. E. Gamboa Saravi, P. Leal Ferreira and F. A. Schaposnik (World Scientific, Singapore, 1990), p. 161; Phys. Lett. B230 (1989), 83; Nucl. Phys. B342 (1990), 680.

[11] P. J. Doust and J. C. Taylor, Phys. Lett. 197B (1987), 232; P. J. Doust, Ann. Phys. (N. Y.) 177 (1987), 169.

[12] G. Leibbrandt, Noncovariant Gauges, World Scientific, Singapore, 1994.

[13] H. Cheng and E. C. Tsai, Phys. Rev. Lett. 57 (1986), 511.

[14] L. Alvarez-Gaumé, J. M. F. Labastida and A. V. Ramallo, Nucl. Phys. B334 (1990), 103; W. Chen, G. W. Semenoff and Y. S. Wu, Mod. Phys. Lett. A5 (1990), 1833 Phys. Rev. D46 (1992), 5521; D. Birmingham, M. Rakowsky and G. Thompson, Phys. Lett. B251 (1990), 121; E. Guadagnini, M. Martellini and M. Mintchev, Phys. Lett. B227 (1989), 111; A. Blasi and R. Collina, Nucl. Phys. B345 (1990), 472; M. Asorey and F. Falceto, Phys. Lett. B241 (1990), 31; F. Delduc, C. Lucchesi, O. Piguet and S. P. Sorella, Nucl. Phys. B346 (1990), 313. 
[15] J. Schwinger, Phys. Rev. 125 (1962), 1043.

[16] Q.-G. Lin and G.-J. Ni, Class. Quantum Grav. 7 (1990), 1261.

[17] G. Dunne, R. Jackiw, S.-Y. Pi and C. A. Trugenberger, Phys. Rev. D 43 (1991), 1332; G. Dunne, Comm. Math. Phys. 150 (1993, 519.

[18] P. Cotta Ramusino, E. Guadagnini, M. Martellini and M. Mintchev, Nucl. Phys. B330 (1990), 557.

[19] F. Ferrari and I. Lazzizzera, Perturbative Analysis of the Chern-Simons Field Theory in the Coulomb Gauge, Preprint UTF 387/96, PAR-LPTHE 96-44. 